Aim of the study: To analyse the diagnostic performance of contrast-enhanced spectral mammography (CESM) based on morphologic and enhancement patterns of mass lesions in dense breast using different protocols: CESM without delayed image and CESM with delayed image.

Material and methods: A total of 151 in formed women with suspicious for malignancy mass lesions in dense breast were included in this study. All of them underwent CESM using 2 protocols. A total of 155 lesions were pathomorphologically verified. We analysed morphologic patterns on low-energy (LE) images and recombined images (RI) by defining the shape, margin, and dynamic patterns based on delayed images.

Results: The comparative analysis revealed that the shape and margins on RI were more significant than those on LE images. The dynamic indicators of CESM were found to be highly significant in dense breasts. The correlation between kinetic curve and histological results demonstrated that a persistent type of curve was common for benign lesions, accounting for $15 / 22$ (68.1\%); plateau and washout - for malignant lesions, accounting for $24 / 89(26.9 \%)$ and $61 / 89(68.5 \%)$, respectively. Delayed image leads to an increase of specificity up to $12.4 \%$, which is statistically significant. The area under the curve (AUC) in CESM with delayed image is larger than that in CESM without delayed image $(p<0.01)$.

Conclusions: CESM is sensitive for the differential diagnosis of breast lesions. CESM with delayed image has higher specificity than CESM without delayed image. Delayed images with plateau and washout are typical for malignancy.

Key words: breast lesions, breast cancer, contrast-enhanced spectral mammography, dense breast.

Contemp Oncol (Pozn) 2021; 25 (1): 17-22 DOI: https://doi.org/10.5114/wo.2021.105030

\section{Contrast-enhanced spectral mammography without and with a delayed image for diagnosing malignancy among mass lesions in dense breast}

\author{
Akmaral Serikovna Ainakulova ${ }^{1,2}$, Zhamilya Zholdybay Zholdybay ${ }^{1}$, \\ Dilyara Radikovna Kaidarova ${ }^{1,2}$, Natalya Igorevna Inozemtceva ${ }^{2}$, \\ Madina Orazaykyzy Gabdullina ${ }^{1}$, Zhanar Kabdualievna Zhakenova ${ }^{1}$, \\ Alexandra Sergeevna Panina ${ }^{1,2}$, Dias Kairatovich Toleshbayev ${ }^{1,2}$, \\ Jandos Mukhtarovich Amankulovi,2
}

${ }^{1}$ Asfendiyarov Kazakh National Medical University, Almaty, Kazakhstan

${ }^{2}$ Kazakh Institute of Oncology and Radiology, Almaty, Kazakhstan

\section{Introduction}

The term density represents an attenuation of $X$-rays at the time they pass through fibro-glandular tissue. The fact that high breast density correlates with high rates of breast cancer is evident now. The prompt diagnosis of breast cancer in women with dense breast is a big challenge for modern radiology. It is generally known that mammography is only partially effective in solving this problem [1]. One of the last studies has identified advantages of breast tomosynthesis compared to full-filled digital mammography for women with dense breasts: the sensitivity for tomosynthesis was found to be significantly higher, reaching $77.4 \%$, but the specificity was almost equal [2]. Contrast-enhanced spectral mammography (CESM) has been identified as a valuable modality in the differential diagnosis of breast lesions [3-7]. The sensitivity of CESM for diagnosing breast cancer has been reported between $90.5 \%$ and $100 \%$ [3, 8-11]. However, its specificity in discriminating malignant from benign lesions is highly varied and has been reported to be in the range $67.9-87.8 \%$ [8, 12-14]. Another study on the clinical performance of CESM in women with breast cancer in dense breast proved that CESM might be comparable to magnetic resonance imaging (MRI) in terms of radiological measurements of mass lesions in dense breasts, and moreover CESM showed less overestimated results than MRI $-16.2 \%$ for CESM and $22.7 \%$ for MRI [15]. Lesions visible in CESM include enhancing masses, non-mass enhancing lesions, and enhancing foci. No specific BI-RADS lexicon for CESM exists. CESM interpretation consists of the BI-RADS assessment based on low-energy (LE) images, which are equal to mammogram and assessment of BI-RADS descriptors for breast MRI. However, this lexicon is based on the evaluation of morphological characteristics of postcontrast enhancement of lesions and has no kinetic curve. Moreover, according to some articles [16, 17], final scoring is subjective. Thus, the final conclusion of CESM consists of the assessment of morphological characteristics of lesions on LE and recombined image (RI) combined. Although the establishment of the kinetic curve may provide new opportunities to maximize CESM accuracy, a literature review shows that there have been only 2 studies with different approaches for this issue $[18,19]$. The purpose of this study was to analyse diagnostic performance of CESM based on morphologic and enhancement patterns of mass lesions in dense breast using different protocols: CESM without and with delayed image. 


\section{Material and methods}

This retrospective study was approved by the local ethics committee. All enrolled patients provided written informed consent in this study.

Participant inclusion criteria: 1) mass lesions suspicious for malignancy (with and without microcalcifications) identified by mammography, ultrasound (US), or both; and 2) heterogeneously dense or extremely dense breast tissue. Participant exclusion criteria were as follows: 1) breast cancer confirmed by nearly done biopsy; 2) breast cancer history; 3) women with breast implant; 4) renal function impairment; 5) pregnancy or lactation; 6) allergic reaction to contrast agent; and 7) hyperthyroidism. Renal function impairment was evaluated by serum creatinine and glomerular filtration rate.

A total of 184 female patients aged 24-78 years (mean $46.9 \pm 10.9$ years) were included in the study during the period from September 2018 to April 2020. All of them had lesions that were suspicious for malignancy or inconclusive results by mammography and US. For the study all of them underwent CESM, which was performed by digital mammograph GE Senographe Essential Full Field Digital System (Mammography X-ray Equipment, Buc, France) using special software. After CESM 28 women with signs of benign lesions on CESM refused biopsy and started 2-year follow-up. They were excluded from the study. Also, there were 5 participants who had a light allergic reaction during CESM and did not finish the examination. They were also excluded. As a result, only 151 women were included in the study.

\section{Contrast-enhanced spectral mammography technique}

For fertile and premenopausal patients, CESM was conducted during the second week of their menstrual cycle, whenever possible; for postmenopausal patients there were no special requirements.

At the time of the study, a trained technologist obtained peripheral intravenous access in the antecubital fossa preferably with a 20 -gauge needle. An iodinated contrast material (1.5 mL/kg dose) was administered intravenously. The catheter remained in place until the end of the examination.

Image acquisition began on the $2^{\text {nd }}$ minute after the beginning of the injection. The examination started with the craniocaudal (CC) view of the breast suspected of malignancy and was followed by the same view of the contralateral breast. Then, mediolateral oblique (MLO) views of both breasts were acquired in the same sequence and completed within 6 minutes. After that, a delayed image of the breast suspected of malignancy was presented only in CC view in order to avoid distortions caused by superimposition effect in MLO views (Fig. 1). As a result, we obtained delayed bilateral (lesions of both breasts) or monolateral (lesions of one breast) images in CC view 6-8 minutes after the initiation of contrast administration. The entire session took 8-10 minutes per patient, including contrast administration.

Low-energy images were performed at the same peak kilovoltage $(\mathrm{kVp})$ and with the same filtration as FFDM, 26-30 kVp. High-energy acquisition was performed with a higher kVp of 45-49. RI were produced by the immediate cancellation of background breast tissue. Only the LE and RE images were sent to the archiving and communication system for interpretation.

After image acquisition CESM without delayed image and CESM with delayed image were blindly reviewed by 2 dedicated, sub-specialized, expert radiologists with more than 5 and 15 years of experience. The assessment of morphologic and enhancement patterns was based on the mammography and MRI lexicon version 5 by the American College of Radiology. On CESM without delayed image: all enhanced lesions considered to be malignant. To analyse CESM with delayed image we used a region of interest (ROI) indicator that was manually placed over the most homogenous area of lesion on RI: CC views on the $2^{\text {nd }}$ and $8^{\text {th }}$ minutes. The diameter of ROI was $2 \mathrm{~mm}$. Based on mean signal in the lesion on RI: CC views on the $2^{\text {nd }}$ and $8^{\text {th }}$ minutes, we distinguished 3 types of contrast accumulation, similar to MR-curve types: 1) persistent - in the case

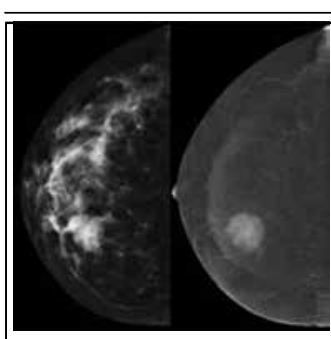

CC suspicious breast

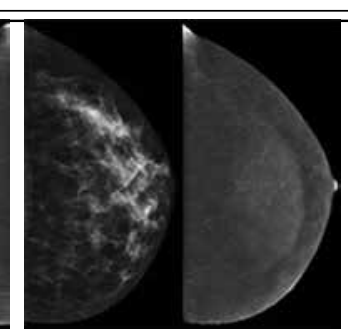

CC normal breast

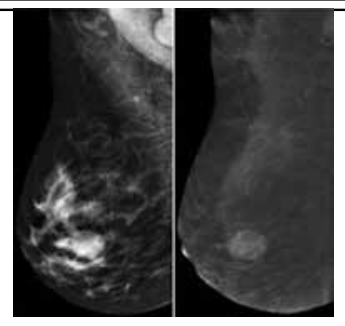

MLO suspicious breast $6^{\text {th }}$ min after contrast administration

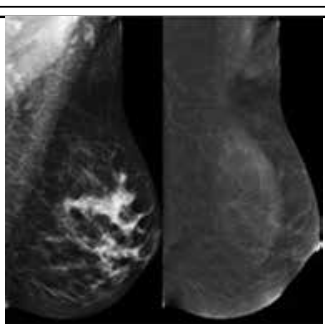

MLO normal breast $8^{\text {th }}$ min after contrast dministration

CESM standard

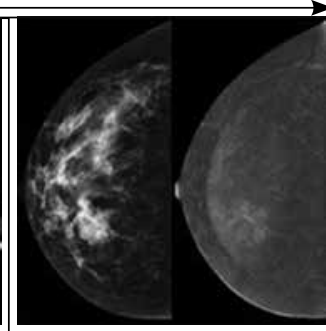

Delayed image suspicious breast

CESM with delayed image

CC-craniocaudal view, MLO - mediolateral oblique view, IV - intravenously

Fig. 1. Schematic performance of the contrast-enhanced spectral mammography technique 

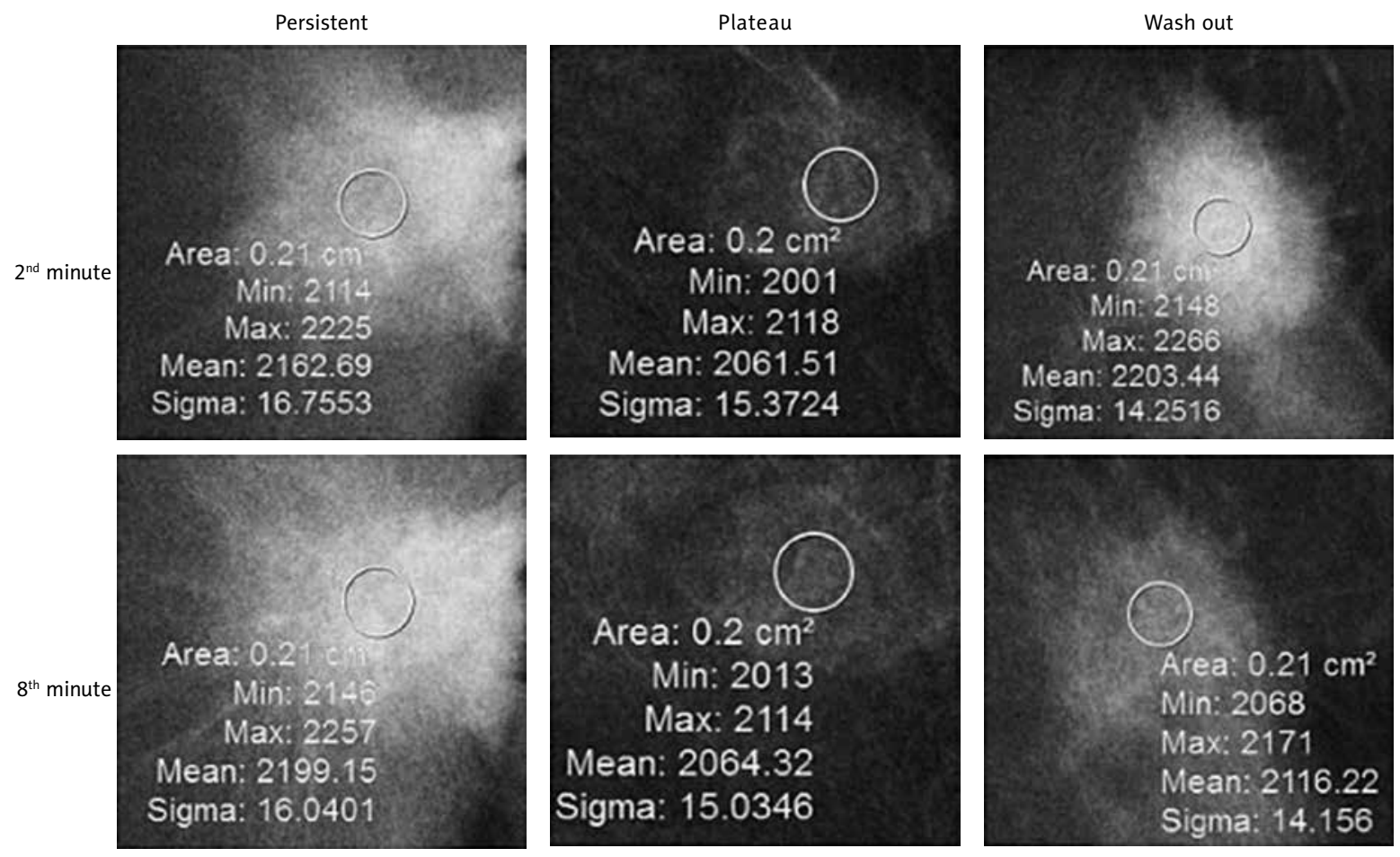

Persistent - increasing the mean signal in the lesion by more than 10 units, plateau - changing the mean signal in the lesion by less than 10 units, washout decreasing the mean signal in the lesion by more than 10 units

Fig. 2. Three types of contrast accumulation in mass lesions

Table 1. Patient characteristics

\begin{tabular}{|c|c|}
\hline \multicolumn{2}{|l|}{ Variable value } \\
\hline $\begin{array}{l}\text { Age (years) } \\
\qquad \begin{array}{l}\text { Mean } \\
\text { Minimum } \\
\text { Maximum }\end{array}\end{array}$ & $\begin{array}{l}46.9 \\
24 \\
78\end{array}$ \\
\hline $\begin{array}{l}\text { Menopausal status }(n, \%) \\
\text { Fertile } \\
\text { Premenopausal } \\
\text { Postmenopausal }\end{array}$ & $\begin{array}{l}37(24.5) \\
59(39.1) \\
55(36.4)\end{array}$ \\
\hline $\begin{array}{l}\text { Breast density }(n, \%) \\
\text { Heterogeneously dense } \\
\text { Extremely dense }\end{array}$ & $\begin{array}{l}102(67.6) \\
49(32.4)\end{array}$ \\
\hline $\begin{array}{l}\text { Breast parenchymal enhancement }(n, \%) \\
\text { Minimal } \\
\text { Mild } \\
\text { Moderate } \\
\text { Marked }\end{array}$ & $\begin{array}{l}12(7.9) \\
104(68.8) \\
31(20.5) \\
4(2.6)\end{array}$ \\
\hline $\begin{array}{l}\text { Lesion localization }(n, \%) \\
\text { Upper inner } \\
\text { Upper outer } \\
\text { Lower inner } \\
\text { Lower outer }\end{array}$ & $\begin{array}{l}22(14.2) \\
104(67.7) \\
22(14.5) \\
7(4.6)\end{array}$ \\
\hline $\begin{array}{l}\text { Lesion size }(n, \%) \\
\text { Less than } 1 \mathrm{~cm} \\
\text { More than } 1.0 \mathrm{~cm} \text { and less than } 2 \mathrm{~cm} \\
\text { More than } 2.0 \mathrm{~cm}\end{array}$ & $\begin{array}{l}27(14.2) \\
59(38.7) \\
69(44.5)\end{array}$ \\
\hline
\end{tabular}

of increasing the mean signal in the lesion by more than 10 units; 2) plateau - in the case of changing the mean signal in the lesion by less than 10 units; and 3) washout - in
Table 2. Pathomorphological analysis of 155 lesions in 151 patients

\begin{tabular}{lclc}
\multicolumn{2}{c}{$\begin{array}{c}\text { Malignant } \\
n=89(57.4 \%)\end{array}$} & \multicolumn{2}{c}{$n=66(42.6 \%)$} \\
DCIS & $8(9 \%)$ & Adenosis & $10(15.2 \%)$ \\
IDC & $69(77.5 \%)$ & Fibroadenoma & $10(15.2 \%)$ \\
ILC & $11(12.4 \%)$ & Fibrocystic changes & $20(30.3 \%)$ \\
Mucinous & $1(1.1 \%)$ & Fibrosis & $12(18.2 \%)$ \\
carcinoma & & Hyperplasia without & $6(9.1 \%)$ \\
& & atypia & \\
& & Mastitis & $3(4.5 \%)$ \\
& & Papilloma & $2(3 \%)$ \\
& & Phyllodes tumour & $3(4.5 \%)$
\end{tabular}

DCIS - ductal carcinoma in situ, IDC - invasive ductal carcinoma, ILC - invasive lobular carcinoma

the case of decreasing the mean signal in the lesion by more than 10 units. Mass lesions with persistent type of kinetic curve were considered to be benign, while those with plateau and washout were considered to be malignant (Fig. 2). Finally, all participants were assigned a BI-RADS-category score of $1-5$ for CESM without delayed image and CESM with delayed image.

\section{Histological analysis}

All patients underwent a biopsy and analysis of post-biopsied material in the Department of Pathomorphology. 
Table 3. Distribution of low-energy image patterns by histopathological results

\begin{tabular}{|c|c|c|c|c|}
\hline \multirow{2}{*}{\multicolumn{2}{|c|}{$\begin{array}{l}\text { Low-energy image } \\
\text { patterns }\end{array}$}} & \multicolumn{2}{|c|}{ Histopathological results } & \multirow[t]{2}{*}{$p$-value } \\
\hline & & Benign (66) & Malignant (89) & \\
\hline \multirow[t]{3}{*}{ Shape } & Oval/lobulated & $26(39.4 \%)$ & 47 (52.8\%) & \multirow[t]{3}{*}{0.057} \\
\hline & Round & $11(16.6 \%)$ & $4(4.5 \%)$ & \\
\hline & Irregular & $29(44.0 \%)$ & $38(42.7 \%)$ & \\
\hline \multirow[t]{5}{*}{ Margins } & Circumscribed & $13(19.7 \%)$ & $13(14.6 \%)$ & \multirow[t]{5}{*}{0.011} \\
\hline & Obscured & 28 (42.4\%) & $20(22.5 \%)$ & \\
\hline & Microlobulated & $7(10.6 \%)$ & $11(12.5 \%)$ & \\
\hline & Indistinct & $18(27.3 \%)$ & 39 (43.7\%) & \\
\hline & Spiculated & 0 & $6(6.7 \%)$ & \\
\hline
\end{tabular}

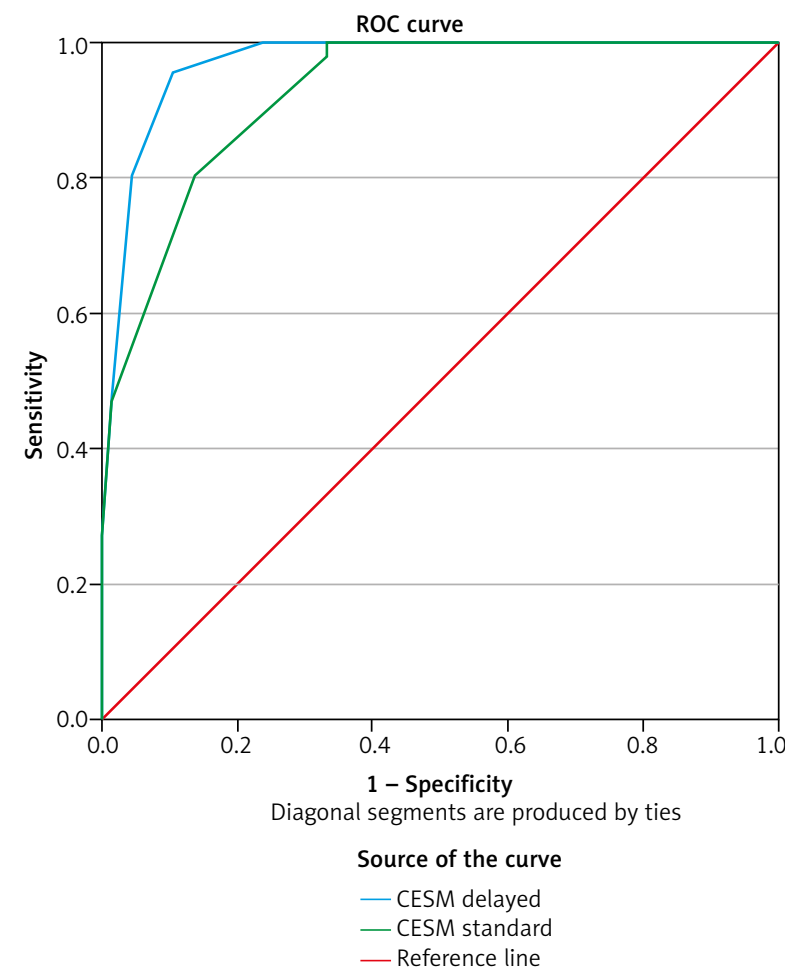

Fig. 3. Receiver operating characteristic (ROC) curve for contrast-enhanced spectral mammography (CESM) with delayed image (blue line) and CESM standard (green line)

A total of 142 (91.6\%) mass lesions were evaluated by US-guided biopsy; stereotactic biopsy was conducted for $7(4.5 \%)$ mass lesions that were invisible on US; and postoperative material was analysed in 6 (3.9\%) cases. Histopathological diagnostics were strictly provided after CESM in order to minimize contrast enhancement in the post-biopsied area and to obtain an accurate assessment of the enhancement type, which is especially crucial for masses that are less than $1.0 \mathrm{~cm}$ in size.

\section{Statistical analysis}

All data were analysed using SPSS ver. 27 software for Windows developed by StatSoft Inc. The $\chi^{2}$ test was applied to assess statistical differences between categorical
Table 4. Distribution of recombined image patterns by histopathological results

\begin{tabular}{|c|c|c|c|c|}
\hline \multicolumn{2}{|c|}{ Subtracted image patterns } & \multicolumn{2}{|c|}{ Histopathological results } & \multirow[t]{2}{*}{$p$-value } \\
\hline & & $\begin{array}{l}\text { Benign } \\
(66)\end{array}$ & $\begin{array}{l}\text { Malignant } \\
\text { (89) }\end{array}$ & \\
\hline \multirow[t]{2}{*}{ Enhancement } & Enhanced & $22^{*}(33.3 \%)$ & 89 (100\%) & \multirow[t]{2}{*}{-} \\
\hline & Non enhanced & $44(66.6 \%)$ & 0 & \\
\hline \multirow[t]{3}{*}{ Shape } & Oval & $14 / 22^{*}(63.7 \%)$ & 39 (43.8\%) & \multirow[t]{3}{*}{0.002} \\
\hline & Round & $5 / 22^{*}(22.7 \%)$ & $5(5.6 \%)$ & \\
\hline & Irregular & $3 / 22^{\star}(13.6 \%)$ & $45(50.6 \%)$ & \\
\hline \multirow[t]{3}{*}{ Margins } & Circumscribed & $12 / 22^{*}(54.5 \%)$ & $13(14.6 \%)$ & \multirow[t]{3}{*}{$<0.001$} \\
\hline & Irregular & $8 / 22^{*}(36.4 \%)$ & $48(54.0 \%)$ & \\
\hline & Spiculated & $2 / 22^{\star}(9.1 \%)$ & 28 (31.4\%) & \\
\hline \multirow{4}{*}{$\begin{array}{l}\text { Internal } \\
\text { enhancement }\end{array}$} & Homogeneous & $13 / 22^{\star}(59.1 \%)$ & $40(45.0 \%)$ & \multirow[t]{4}{*}{0.001} \\
\hline & Heterogeneous & $6 / 22^{\star}(27.3 \%)$ & $49(55.0 \%)$ & \\
\hline & $\begin{array}{l}\text { Rim } \\
\text { enhancement }\end{array}$ & $1 / 22^{\star}(4.5 \%)$ & 0 & \\
\hline & $\begin{array}{l}\text { Dark internal } \\
\text { septation }\end{array}$ & $2 / 22^{\star}(9.1 \%)$ & 0 & \\
\hline \multirow{3}{*}{$\begin{array}{l}\text { Dynamic } \\
\text { kinetic curve }\end{array}$} & Persistent & $15 / 22^{\star}(68.2 \%)$ & $4(4.5 \%)$ & \multirow[t]{3}{*}{$<0.001$} \\
\hline & Plateau & $6 / 22^{\star}(27.3 \%)$ & $24(27.0 \%)$ & \\
\hline & Washout & $1 / 22^{*}(4.5 \%)$ & $61(68.5 \%)$ & \\
\hline
\end{tabular}

patterns among malignant and benign lesions. The cross tabulations in dichotomized patterns were used to calculate accuracy, sensitivity, specificity, positive predictive value, and negative predictive value. $P$-values $<0.05$ were considered statistically significant.

\section{Results}

Out of 151 females, 102 (67.5\%) had heterogeneously dense and 49 (32.5\%) had extremely dense breast tissue; 104 (68.9\%) patients had mild breast parenchymal enhancement; 104 (67.7\%) lesions were located in the upper-outer quadrant (Table 1 ).

The quantity of lesions was 155, because 4 women had bilateral process. Among 155 lesions, 111 (71.6\%) enhanced the contrast agent. Eighty-nine (57.4\%) of them were histologically confirmed with malignancy, but 22 (14.2\%) were benign. Forty-four (28.4\%) lesions did not have contrast enhancement and were also pathomorphologically confirmed to be benign; hence, the absolute number of benign lesions was 66 (42.6\%). All malignant lesions were performed with mass lesions and masses with calcification, 34 women were diagnosed with multifocal/multicentric processes. The distribution of malignant and benign lesions is shown in Table 2.

Tables 3 and 4 demonstrate morphological patterns in LE images and RI (respectively) in relation to the pathomorphological analysis. The lesion shape pattern on LE images was found to be non-significant ( $p=0.057$ ), unlike 
the lesion margin pattern, which was found to be highly significant $(p=0.011)$. RI led to improvement of the significance of shape and margins, amounting to $p=0.002$ and $p<0.001$, respectively. Irregular shape, and irregular and spiculated margins were presented mostly in malignant lesions.

The dynamic indicators of CESM with delayed image were determined to be highly significant. All 89 (100\%) malignant lesions had enhancement on RI, and 44/66 $(66.6 \%)$ benign lesions had no enhancement and were considered to be truly negative lesions $(p<0.001)$. The correlation between kinetic curve and histological results demonstrated that a persistent type of curve was common for benign lesions, accounting for 15/22 (68.1\%); plateau and washout patterns were common for malignant lesions, accounting for 24/89 (26.9\%) and 61/89 (68.5\%), respectively.

Table 5 compares diagnostic performance characteristics in CESM without and CESM with delayed image. Delayed image leads to an increase in specificity of up to $12.4 \%$, which is statistically significant. The area under the curve (AUC) of ROC in CESM with delayed image is larger than that in CESM without delayed image ( $p<$ 0.01) (Fig. 3).

The dynamic kinetic curve analysis (Table 4) of 22 benign lesions detected a persistent type of curve in 15 $(68.2 \%)$ lesions, plateau in $6(27.3 \%)$ lesions, and washout in $1(4.5 \%)$ case. There were $4(2.6 \%)$ false negatives among 155 lesions, which had persistent curve type (sensitivity 93.7\%) (Table 5). All of them were ductal carcinoma in situ (DCIS). Three of the remaining DCIS were invisible in LE images but enhanced contrast on RI, and 2 were visible on LE images but enhanced contrast only on delayed images. There were 7 (4.5\%) false positive assessments on CESM with delayed image, 6 of them had plateau and 1 had washout (specificity 92.4\%). A total of 3 cases of Phyllodes tumour and 3 fibroadenomas were related to plateau. The case of washout was confirmed with mastitis. The accuracy rate was $92.4 \%$ (Table 5).

\section{Discussion}

In view of the fact that density is accepted as one of the most important breast cancer predictors, breast cancer detection in the female Asian population with dense breasts is becoming the most critical issue for radiologists [20]. Mammography is known to be particularly effective for this purpose [1]. Ultrasound is good as a supplementary option to detail lesion features detected by mammography [21-23]. Magnetic resonance imaging of the breast is known as a gold standard of breast cancer detection due to its ability to reveal pathological neovascularity, which is historically associated with malignancy [21, 24]. However, it is apparent that benign lesions often enhance, causing insufficient specificity $[25,26]$. The other challenge for using MRI is local inaccessibility and high cost [27, 28]. CESM is fundamentally identical to breast MRI in principle, and it has been reported to have a high rate of sensitivity but medium rate of specificity in the literature [3, 29, 30], which is caused by masking effects of the overlap of dense
Table 5. Diagnostic performance characteristics of contrast-enhanced spectral mammography without delayed image and contrast-enhanced spectral mammography with delayed image

\begin{tabular}{|c|c|c|}
\hline & CESM standard & CESM with delayed image \\
\hline Sensitivity (\%) ${ }^{a}$ & 97.8 & 93.7 \\
\hline Specificity (\%) & 80.0 & 92.4 \\
\hline PPV $(\%)^{a}$ & 66.7 & 89.4 \\
\hline NPV $(\%)^{a}$ & 98.9 & 95.5 \\
\hline Accuracy (\%) & 64.27 & 92.9 \\
\hline$A \cup C^{b}$ & $\begin{array}{c}0.924 \\
(0.883-0.964)\end{array}$ & $\begin{array}{c}0.969 \\
(0.942-0.995)\end{array}$ \\
\hline$p$-value & & $<0.001$ \\
\hline
\end{tabular}

a - values in parentheses are raw numbers used to calculate percentages, ${ }^{b}$ - values in parentheses are $95 \% \mathrm{Cl}, \mathrm{PPV}$ - positive predictive value, $N P V$ - negative predictive value, AUC - area under the curve

breast tissue. Hence it creates a demand for the development of new applications of existing methods.

Considering the ease of access and cost-effectiveness of CESM, we devoted this study to analysing the diagnostic performance of CESM based on morphological and enhancement patterns of mass lesions in dense breasts. We compared CESM patterns acquired by different protocols: CESM without and CESM with delayed images.

The goal of this study was to approach the quality of breast MRI. Thus, we integrated delayed images up to the $8^{\text {th }}$ minute after contrast agent administration to acquire the kinetic curve based on the mean signal difference at the $2^{\text {nd }}$ minute and $8^{\text {th }}$ minute after the injection.

Using the ROC analysis, we determined that CESM with delayed image leads to improvement of specificity (AUC greater than 0.3). The persistent type of curve was typical for benign lesions, while malignant ones had plateau and washout.

The limitation of this study is the fact that we included only masses and did not analyse architectural distortion, asymmetry, and calcifications. Another limitation is that within the study, we discussed the likelihood of malignancy among mass lesions based on the contrast agent enhancement type. In the future, it would be reasonable to integrate the morphological and dynamic criteria to assess final category BI-RADS and explore disparities between breast MRI and CESM in malignant lesions.

\section{Conclusions}

CESM is sensitive for the differential diagnosis of breast lesions. CESM with delayed image has higher specificity than CESM without delayed image. Delayed images with plateau and washout are typical for malignancy. The assessment of mass morphologic features in combination with dynamic kinetic patterns of contrast enhancement will lead to significant improvement in the accuracy of CESM.

The authors declare no conflict of interest. 


\section{References}

1. Choi WJ, Cha JH, Kim HH, Shin HJ, Chae EY. Analysis of prior mammography with negative result in women with interval breast cancer. Breast Cancer 2016; 23: 583-589.

2. Babkina TM, Dykan IM, Gurando AV, Suleimenova DM, Kozarenko TM, Bozhok YM, Stuley VA. Detection of breast cancer presenting as a mass in women with dense breasts - digital breast tomosynthesis versus full-field digital mammography. Exp Oncol 2020; 42: 215-219

3. Łuczyńska E, Heinze-Paluchowska S, Hendrick E, et al. Comparison between breast MRI and contrast-enhanced spectral mammography. Med Sci Monit 2015; 21: 1358-1367.

4. Xing D, Yongbin L, Sun B, et al. Diagnostic value of contrast-enhanced spectral mammography in comparison to magnetic resonance imaging in breast lesions. J Comput Assist Tomogr 2019; 43 245-251.

5. Travieso-Aja M, Maldonado-Saluzzi D, Naranjo-Santana P, et al. Diagnostic performance of contrast-enhanced dual-energy spectral mammography (CESM): a retrospective study involving 644 breast lesions. Radiol Med 2019; 124: 1006-1017.

6. Luczyńska E, Heinze-Paluchowska S, Dyczek S, Blecharz P, Rys J, Reinfuss M. Contrast-enhanced spectral mammography: comparison with conventional mammography and histopathology in 152 wom en. Korean J Radiol 2014; 15: 689-696.

7. Tennant SL, James JJ, Cornford EJ, et al. Contrast-enhanced spectral mammography improves diagnostic accuracy in the symptomatic setting. Clin Radiol 2016; 71: 1148-1155.

8. Sorin V, Yagil Y, Yosepovich A, Shalmon A, Gotlieb M, Neiman OH, Girio-Fragkoulakis C. Contrast-Enhanced Spectral Mammography in Women with Intermediate Breast Cancer Risk and Dense Breasts. AJR Am J Roentgenol 2018; 211: 267-274.

9. Lalji UC, Houben IP, Prevos R, et al. Contrast-enhanced spectral mammography in recalls from the Dutch breast cancer screening program: validation of results in a large multireader, multicase study. Eur Radiol 2016; 26: 4371-4379.

10. Perek S, Kiryati N, Zimmerman-Moreno G, Sklair-Levy M, Konen E, Mayer A. Classification of contrast-enhanced spectral mammogra phy (CESM) images. Int J Comput Assist Radiol Surg 2019; 14: 249-257.

11. Łuczyńska E, Niemiec J, Hendrick E, et al. Degree of enhancement on contrast enhanced spectral mammography (CESM) and lesion type on mammography (MG): comparison based on histological results. Med Sci Monit 2016; 22: 3886-3893.

12. Cheung YC, Lin YC, Wan YL, et al. Diagnostic performance of dual-energy contrast-enhanced subtracted mammography in dense breasts compared to mammography alone: interobserver blind-reading analysis. Eur Radiol 2014; 24: 2394-2403.

13. Cheung YC, Tsai HP, Lo YF, et al. Clinical utility of dual energy contrast-enhanced spectral mammography for breast microcalcifica tions without associated mass: a preliminary analysis. Eur Radiol 2016; 26: 1082-1089.

14. Lewin JM, Isaacs PK, Vance V, Larke FJ. Dual-energy contrast-enhanced digital subtraction mammography: feasibility. Radiol 2003; 229: 261-268

15. Bozzini A, Nicosia L, Pruneri G, et al. Clinical performance of contrast-enhanced spectral mammography in pre-surgical evaluation of breast malignant lesions in dense breasts: a single center study. Breast Cancer Res Treat 2020; 184: 723-731.

16. Chi X, Zhang L, Xing D, Gong P, Chen Q, LV Y. Diagnostic value of the enhancement intensity and enhancement pattern of CESM to benign and malignant breast lesions. Medicine (Baltimore) 2020; 99: e22097

17. Gity M, Arabkheradmand A, Taheri E, Shakiba M. Diagnostic investigation of breast magnetic resonance imaging in malignant and benign mass lesions. Arch Med Sci 2018; 14: 1061-1069.

18. Rudnicki W, Heinze S, Niemiec J, Kojs Z, Sas-Korczynska B, Hendrick E, Luczynska E. Correlation between quantitative assessment of contrast enhancement in contrast-enhanced spectral mammography (CESM) and histopathology-preliminary results. Eur Radiol 2019; 29: 6220-6226.
19. Deng CY, Juan YH, Cheung YC, et al. Quantitative analysis of enhanced malignant and benign lesions on contrast-enhanced spectral mammography. Br J Radiol 2018; 91: 20170605.

20. Bae JM, Kim EH. Breast density and risk of breast cancer in asian women: a meta-analysis of observational studies. J Prev Med Public Health 2016; 49: 367-375.

21. Gilbert FJ, Pinker-Domenig K. Diagnosis and Staging of Breast Cancer: When and How to Use Mammography, Tomosynthesis, Ultrasound, Contrast-Enhanced Mammography, and Magnetic Resonance Imaging. In: Hodler J, Kubik-Huch R, von Schulthess G (eds.). Diseases of the Chest, Breast, Heart and Vessels 2019-2022: Diagnostic and Interventional Imaging, $1^{\text {st }}$ ed. Springer, California 2019: 155-166.

22. Burkett BJ, Hanemann CW. A review of supplemental screening ultrasound for breast cancer: certain populations of women with dense breast tissue may benefit. Acad Radiol 2016; 23: 1604-1609.

23. Rebolj M, Assi V, Brentnall A, Parmar D, Duffy SW. Addition of ultrasound to mammography in the case of dense breast tissue: systematic review and meta-analysis. Br J Cancer 2018; 118: 1559-1570.

24. Messineo D, Izzo L, Pisanelli MC, Razionale F, Izzo S, Izzo P. The influence of preoperative MRI in early breast cancer: gold standard. Ann Ital Chir 2020; 91: 144-153.

25. Jochelson MS, Dershaw DD, Sung JS, et al. Bilateral contrast-enhanced dual-energy digital mammography: feasibility and comparison with conventional digital mammography and MR imaging in women with known breast carcinoma. Radiology 2013; 266: 743-751.

26. Badr S, Laurent N, Régis C, Boulanger L, Lemaille S, Poncelet E. Dual-energy contrast-enhanced digital mammography in routine clinical practice in 2013. Diagn Interv Imaging 2014; 95: 245-258.

27. Patel BK, Gray RJ, Pockaj BA. Potential cost savings of contrast-enhanced digital mammography. AJR Am J Roentgenol 2017; 208: 231-237.

28. Phillips J, Steinkeler J, Talati K, et al. Workflow considerations for incorporation of contrast-enhanced spectral mammography into a breast imaging practice. J Am Coll Radiol 2018; 15: 881-885.

29. Li L, Roth R, Germaine P, et al. Contrast-enhanced spectral mammography (CESM) versus breast magnetic resonance imaging (MRI): a retrospective comparison in 66 breast lesions. Diagn Interv Imaging 2017; 98: 113-123.

30. Fallenberg EM, Dromain C, Diekmann F, et al. Contrast-enhanced spectral mammography versus MRI: initial results in the detection of breast cancer and assessment of tumour size. Eur Radiol 2014; 24: $256-264$

\section{Address for correspondence}

\section{PhD-candidate Madina Orazaykyzy Gabdullina}

Asfendiyarov Kazakh National University

94 Tole bi St.

Almaty, Kazakhstan

e-mail: madina.orazaykyzy@gmail.com

\section{PhD-candidate Akmaral Serikovna Ainakulova}

Asfendiyarov Kazakh National University

94 Tole bi St.

Almaty, Kazakhstan

email: ar89@list.ru

Submitted: 27.012021

Accepted: 27.02.202 